\title{
Rare case of a recurrent juvenile ossifying fibroma of the lumbosacral spine
}

\author{
Cecilia L. Dalle Ore, BA, ${ }^{1}$ Darryl Lau, MD, ${ }^{1}$ Jessica L. Davis, MD, ${ }^{2}$ Michael M. Safaee, MD, ${ }^{1}$ and \\ Christopher P. Ames, MD'1
}

\author{
Departments of ${ }^{1}$ Neurological Surgery and ${ }^{2}$ Pathology, University of California, San Francisco, California
}

\begin{abstract}
Juvenile ossifying fibroma (JOF) is a rare benign bone tumor that occurs most frequently in the craniofacial bones of children and young adults. There are few case reports that describe its involvement outside the craniofacial skeleton, especially within the spinal column. While JOF is classified as a benign lesion, it may be locally aggressive and demonstrate a high propensity for recurrence, even after resection. Definitive surgical management may be challenging in naive cases, but it is particularly challenging in recurrent cases and when extensive spinal reconstruction is warranted. In this report, the authors describe the diagnosis and surgical management of a 29-year-old man who presented with a large recurrent sacral trabecular-subtype JOF. A review of literature regarding JOFs, management of recurrent primary spinal tumors, and sacral reconstruction are discussed.
\end{abstract}

https://thejns.org/doi/abs/10.3171/2017.10.SPINE17947

KEY WORDS en bloc resection; intralesional resection; juvenile ossifying fibroma; recurrent; sacrectomy; spinal tumor; oncology

$\mathbf{J}$ UVENILE ossifying fibroma (JOF) is a rare benign tumor that typically occurs in the craniofacial bones of children and young adults. ${ }^{13,14}$ There are only 2 published cases of JOF or ossifying fibroma involving the spinal column, ${ }^{29,44}$ and there are no reports in the literature of a recurrent ossifying fibroma involving the spinal column. While malignant transformation of these tumors has not been described, they can grow rapidly and display locally aggressive behavior, causing destruction of surrounding structures. ${ }^{13} \mathrm{~A}$ high propensity for local recurrence necessitates complete and of ten en bloc resection..$^{13,42}$ While surgical intervention is the definitive treatment for JOF, complete resection of these tumors located within the spinal column presents substantial technical challenges due to the proximity of nervous and vascular structures and need for spinal reconstruction. In this report, we describe the case of a patient who presented with a recurrent sacral trabecular-subtype JOF and underwent intralesional resection of the tumor and sacral reconstruction (sacroplasty). In addition, we review the literature regarding JOF involving the spine and discuss the surgical management of recurrent locally aggressive bony tumors involving the spinal column.

\section{Case Report}

History and Presentation

A 29-year-old man with a history of a previously resected L-5 primary tumor presented with new back and leg pain. He had undergone an L-5 vertebrectomy, anterior column cage reconstruction, and L4-S1 posterior spinal fixation at an outside institution 2 years earlier. Based on histopathological findings at that time, he had been diagnosed as having a desmoplastic fibroma. On presentation to us, he reported 3 weeks of new back and leg pain. $\mathrm{He}$ had been using a back brace, narcotics, and gabapentin without relief of his pain, which was quite debilitating and greatly limited his mobility. He did not have bowel or urinary issues. Findings from motor and sensory examinations were normal.

\section{Imaging Findings}

Radiographs demonstrated his prior L-5 vertebrectomy and L4-S1 posterior spinal fusion (Fig. 1). CT scanning of his lumbosacral spine demonstrated solid fusion, but with a new $7.7 \times 4.1 \times 6.7-\mathrm{cm}$ complex lytic lesion involving the sacrum, resulting in near-complete destruction of the left

ABBREVIATIONS EBL = estimated blood loss; JOF = juvenile ossifying fibroma; JPOF = juvenile psammomatoid ossifying fibroma; JTOF = juvenile trabecular ossifying fibroma; MMA = methyl methacrylate. 


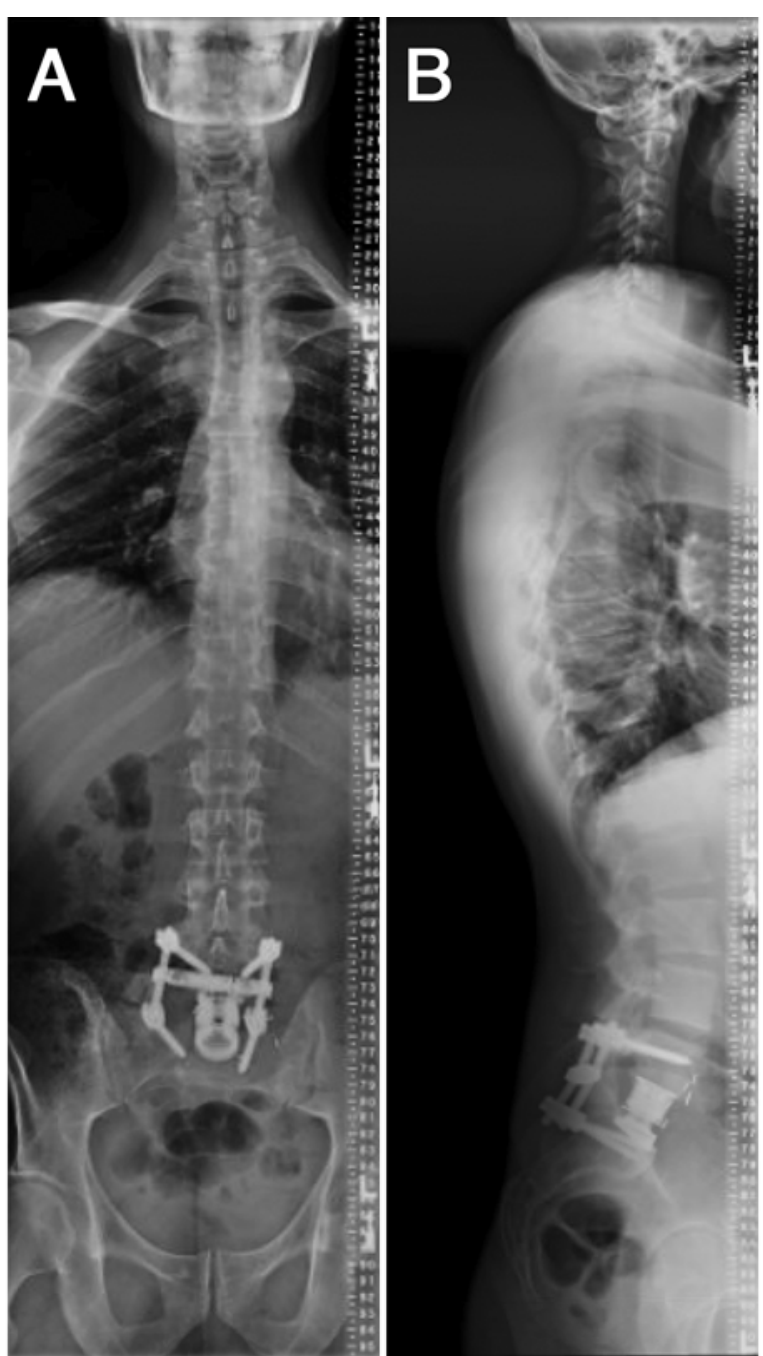

FIG. 1. Preoperative anteroposterior $(\mathbf{A})$ and lateral $(\mathbf{B})$ radiographs showing the preexisting corpectomy cage and L4-S1 posterior spinal fusion.

sacral ala (Fig. 2). There was also a nondisplaced pathologic fracture through the anterior portion of the sacrum. MRI demonstrated minimal homogeneous enhancement of the tumor. The tumor encroached into the central canal
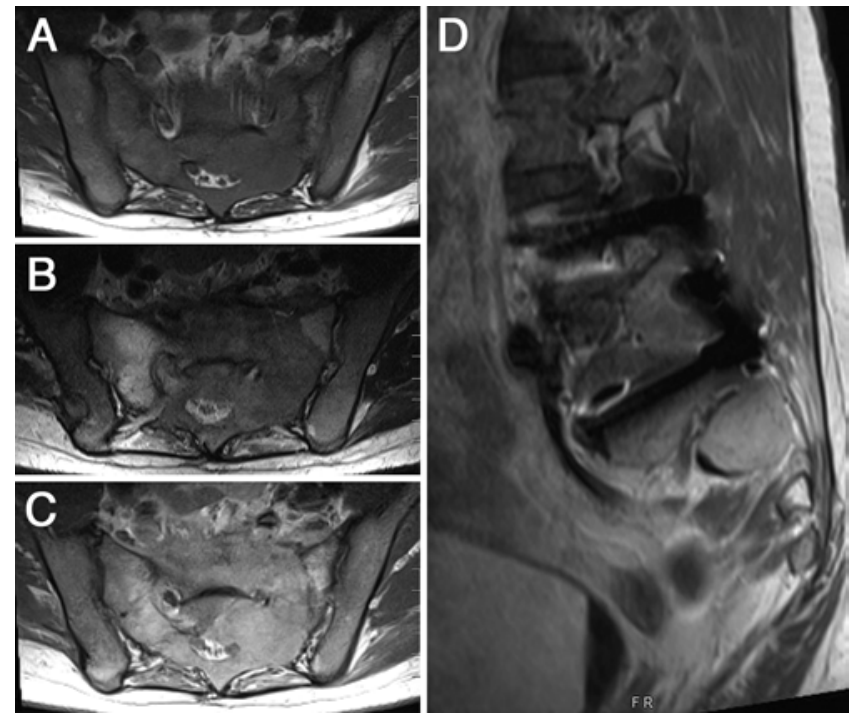

FIG. 3. A-C: Axial T1-weighted (A), T1-weighted Gd-enhanced (B), and T2-weighted (C) MR images obtained at the level of S-1, showing the recurrent sacral tumor with minimal heterogeneous enhancement. D: Sagittal T2-weighted image showing the recurrent tumor encroaching into the central canal at S-1 and encasing the exiting left S-1 nerve root.

at the level of S-1 and encased the exiting left S-1 nerve root (Fig. 3).

\section{Tumor Resection and Sacral Reconstruction}

The patient was taken to the operating room for planned single-stage hardware removal, tumor removal via intralesional resection, partial sacrectomy with sacroplasty, and posterior spinal instrumentation/fusion. The patient was positioned supine, and a midline incision was made from L-3 down to the pelvis. The previously placed hardware was encountered at the L-4 and S-1 levels; the fusion at L4-5 was noted to be solid, and there was no loosening of any screws. L-3 pedicle screws were placed, and the old pedicle screws at L-4 were removed and replaced with larger-diameter screws. Given the patient's pelvic insufficiency fracture due to tumor destruction, 2 iliac screws were placed bilaterally. Laminectomies were then performed from L-4 to S-4. A large amount of tumor was encountered that was densely adherent to the dura. The dor-
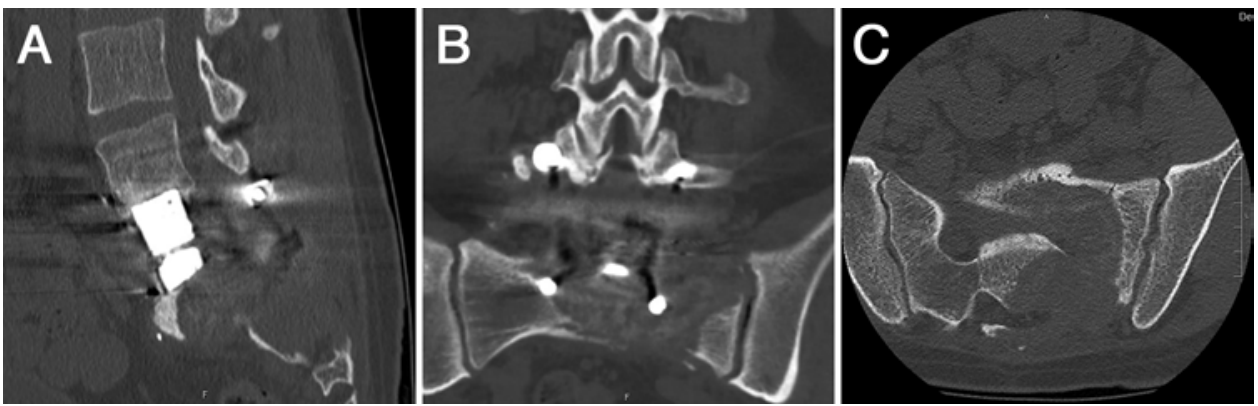

FIG. 2. A: Sagittal lumbar CT scan demonstrating an intact corpectomy cage with a lytic lesion inferior and posterior to the preexisting construct. B and C: Coronal CT scans of the lumbar spine showing a new $7.7 \times 4.1 \times 6.7-\mathrm{cm}$ complex lytic sacral lesion with near-complete destruction of the left sacral ala. 


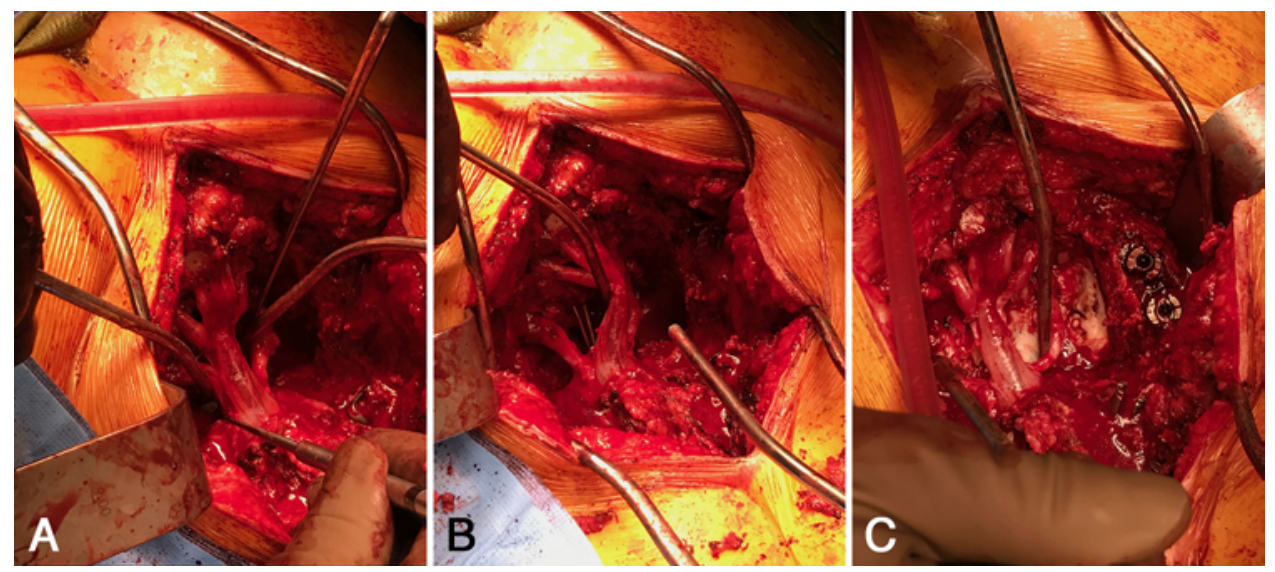

FIG. 4. Intraoperative images. A: Following the wide decompression and complete S-1 corpectomy for tumor resection, a large defect is seen. B: The K-wires are secured in place to facilitate reconstruction of the sacrum. C: The final sacroplasty construct. Figure is available in color online only.

sal aspect of the tumor was carefully dissected off the dura and removed. The sacral laminectomy was then expanded laterally, exposing the sacral nerve roots. The S-1 pedicles were skeletonized and removed to decompress the ventral surface of the sacral nerve roots. An S-1 corpectomy was carried out until normal bony endplates were encountered. During tumor resection, bony margins were sent for histological analysis to ensure that no viable tumor was present. Resection produced a large sacral defect extending from the bottom of the prior L-5 cage to the mid-sacrum (Fig. 4A). The sacral defect was reconstructed using a cement bar technique. Multiple K-wires were placed in the sacral defect (Fig. 4B) and methyl methacrylate (MMA) was introduced to reconstruct the sacrum (Fig. 4C). Cobalt chrome rods were secured in place and the plastic surgery team closed the wound. Estimated blood loss (EBL) was 6 L. There were no intraoperative complications.

\section{Histopathological Examination}

Hematoxylin and eosin-stained sections demonstrated a fibro-osseous proliferation composed of variably cellular spindled cells arranged in a fascicular to vaguely storiform pattern embedded with numerous anastomosing trabeculae of osteoid and woven bone (Fig. 5A). The bony trabeculae had prominent osteoblastic rimming (Fig. 5A and B). The spindled stroma was bland, without cytological atypia, and ranged from hypercellular to loose, edematous areas to mature/collagenized stroma (Fig. 5C). Focal hemorrhage and clusters of multinucleate, osteoclasticlike giant cells were present (Fig. 5C). Inconspicuous mitoses were present (up to 3 mitoses per $10 \mathrm{hpf}$ ) and no necrosis was present. The majority of the tumor showed marked trabecular osteoid and woven bone; however, a rare focus demonstrated an abrupt transition to rounded, psammomatoid ossicles of calcification (more characteristic of conventional ossifying fibroma; Fig. 5D). Immunohistochemical staining for MDM2 and CDK4 were performed and were negative in the spindle-shaped cells; this in conjunction with the morphology and lack of cytological atypia argued against this lesion representing a lowgrade osteosarcoma. Overall, the morphological features were that of a juvenile ossifying fibroma and specifically of a juvenile trabecular ossifying fibroma.

\section{Complications}

The patient had no perioperative complications and was at his neurological baseline at the time of discharge to home. His postoperative images are shown in Fig. 6. Five weeks after surgery, the patient presented to the emergency department with worsening back pain and new radicular leg pain. There were no abnormalities on neurological examination. CT and MRI revealed evidence of a fluid collection within the surgical cavity concerning for sterile seroma versus infection. No bony fractures or hardware failure were identified. The fluid collection was tapped under CT guidance and the fluid was consistent with old blood.

The patient presented again after CT-guided drainage with a new left foot drop. On examination, his anterior tibialis muscle strength on the left was $1 / 5$ and extensor hallucis longus muscle strength was $2 / 5$. In addition, exploratory reoperation revealed an organized hematoma on the left, surrounding the L-5 nerve root and lateral portion of the dura. After evacuation of the hematoma, intraoperative neuromonitoring demonstrated a $40 \%$ improvement in motor evoked potentials in the left tibialis anterior and extensor hallucis longus muscles. The implants were not manipulated, and the bone graft was not disturbed. There was no evidence of purulence. Fluid from the surgical cavity was collected for culture, which grew rare gram-positive rods in a delayed fashion that were speciated to Mycobacterium fortuitum. The patient was treated with 2 months of parenteral amikacin and 1 year of oral clarithromycin and moxifloxacin. His foot drop improved postoperatively, and at the time of discharge he had 4/5 strength in both the anterior tibialis and extensor hallucis longus muscles. At the 6-month follow-up, the patient's foot drop had completely resolved. The patient was to return for follow-up every 6 months for 2 years following his index surgery, and yearly thereafter.

\section{Discussion}

JOF is a rare benign fibro-osseous lesion that typically 

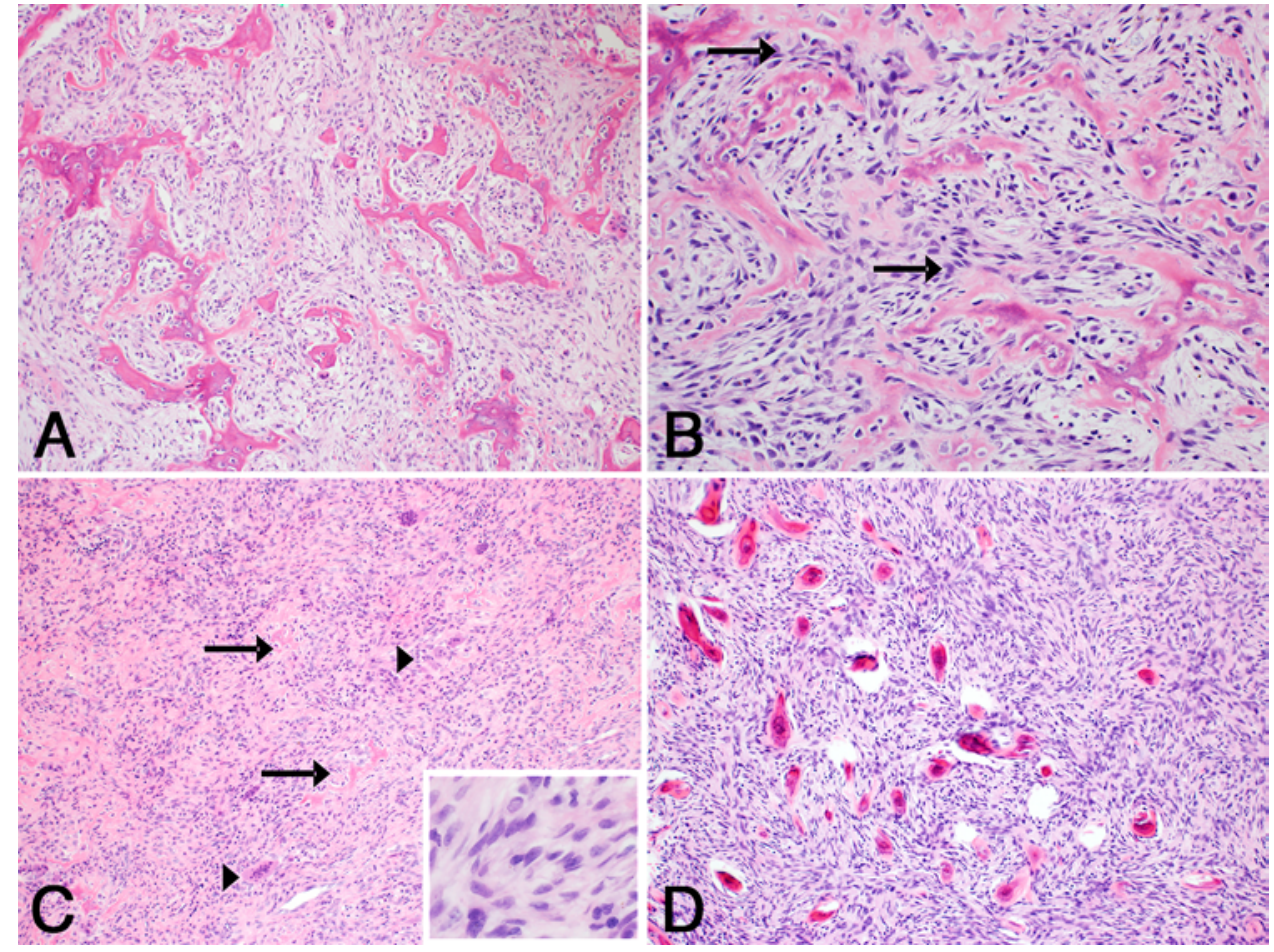

FIG. 5. Histopathological examination. A: Image showing a fibro-osseous lesion composed of loose fibrous stroma and numerous anastomosing trabeculae of woven bone with prominent osteoblastic rimming. B: Trabecular woven bone with markedly prominent osteoblastic rimming. There are numerous plump cells with eccentric nuclei and amphophilic cytoplasm that line the woven bone and extend between the bony trabeculae are osteoblasts (arrows). C: Bland spindle cell stroma with clusters of multinucleate, osteoclastic giant cells (arrowheads), and scattered areas of osteoid/woven bone (arrows). Fibroblastic stroma without atypia (inset). D: Fibroblastic stroma in a storiform arrangement with embedded round "psammomatoid" ossicles of calcification. $\mathrm{H} \& \mathrm{E}$, original magnification $\times 100(A, C$, and D); $\times 200$ (B); ×400 (inset). Figure is available in color online only.

arises in the craniofacial bones of 4- to 15-year-old children, although it can also occur in adults. The mean age at diagnosis of JOF is 11 years, with no sex predilection. Tumors usually involve the facial bones, most commonly the maxilla and mandible (85\% of cases), and less frequently the sinonasal bones and the calvaria. ${ }^{18,28,32,37,40}$ Presentation of JOF and other ossifying fibromas outside of the craniofacial skeleton is unusual, with only one prior case of JOF and one prior adult ossifying fibroma previously reported in the spine. ${ }^{44}$ Uslu et al. described the subtotal resection of a JOF occurring in the lumbar spine of a 16-year-old boy; imaging at 18 months demonstrated residual tumor. ${ }^{44}$ Similarly, Ohyama et al. described the subtotal resection of an ossifying fibroma in the thoracolumbar spine of a 43-year-old woman. ${ }^{29}$ At 18 months, there was no clinical or radiographic evidence of disease. ${ }^{29}$ Patients with JOF may present with pain and local deformity; however, the majority of patients are asymptomatic.

On imaging, JOFs present as radiolucent, unilocular or multilocular lesions with well-defined to sclerotic borders that gradually and variably become radiopaque due to calcification..$^{35,37,44}$ In our case, the lesion had well-defined borders on CT imaging but no distinct calcifications. JOFs might show subtle contrast enhancement on CT and demonstrate low to hypointense signal on T2-weighted MRI. ${ }^{10}$ Given the variable imaging presentation of the entity and difficulty in distinguishing JOF from other more common primary bony lesions (including fibrous dysplasia, central giant cell tumor, or rarely a more aggressive lesion such as osteosarcoma), histological examination of tumor specimens is necessary for definitive diagnosis, particularly in areas outside the craniofacial skeleton. Pathologically, the differential diagnosis of JOF includes other fibro-osseous lesions, primarily fibrous dysplasia when occurring in craniofacial sites; at unusual sites, considerations would include osteofibrous dysplasia or low-grade central osteosarcoma. ${ }^{14}$

Our patient had been previously diagnosed with a fibroblastic proliferation suggestive of a desmoplastic fibroma; however, rare foci of bone were noted within the lesion, which would be unusual for desmoplastic fibroma. ${ }^{17,38} \mathrm{JOF}$ is a fibro-osseous lesion characterized by cellular bland fibroblastic stroma with embedded trabeculae and/or ossicles of osteoid and woven bone. ${ }^{13}$ Two clinicopathologic subtypes of JOF have been described: juvenile trabecular ossifying fibroma (JTOF) and juvenile psammomatoid ossifying fibroma (JPOF)., ${ }^{7,13,14,39,40}$ Both forms are clinically characterized by rapid growth and more frequent recurrences than conventional ossifying fibroma: recurrence of both JTOF and JPOF has been documented in 30\%-50\% of cases, necessitating long-term follow-up. ${ }^{18,47}$

JPOF is histologically characterized by multiple small calcified ossicles (resembling psammoma bodies) embedded within bland fibrous stroma. ${ }^{13,14}$ JTOF is characterized by anastomosing trabeculae of immature osteoid and woven bone rimmed with variably prominent osteoblasts. ${ }^{13}$ 


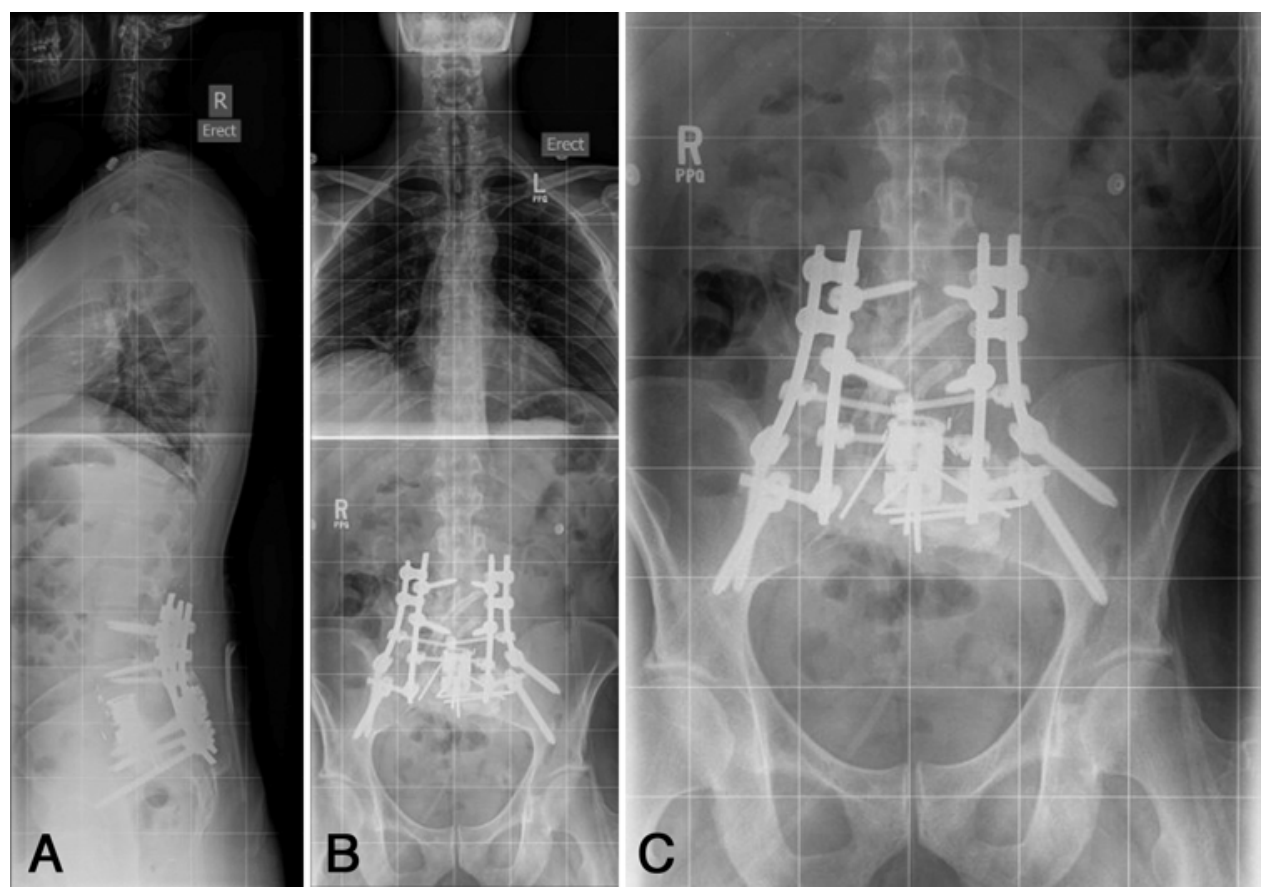

FIG. 6. Postoperative radiographs demonstrating the final spinal construct from L-3 to the pelvis. A and B: Postoperative standing 36-inch lateral (A) and anteroposterior (B) radiographs. Normal regional and global spinal alignment is demonstrated. More importantly, an intact construct is seen, consisting of the sacroplasty with the prior corpectomy cage and new fusion construct consisting of L3-4 bilateral pedicle screws and double bilateral iliac screws (4 total) secured with double bilateral rods (4 total) and 2 cross-links. C: Close-up anteroposterior view of the construct.

The fibroblastic stromal cells present in both JPOF and JTOF are spindled to stellate with bland cytomorphology; occasional mitoses may be seen but not atypical forms. ${ }^{13}$ Clusters of osteoclast-like giant cells are often identified, in particular in areas of hemorrhage, and, rarely, secondary aneurysmal bone cyst change may be observed. ${ }^{13}$ JOFs are unencapsulated tumors and, while they are typically well delineated, often by rind of reactive/woven bone, they can be infiltrative and/or destructive to the surrounding bone. ${ }^{13}$

Due to the known natural history of high risk for recurrence and locally aggressive behavior, the first line of treatment for JOF is complete resection..$^{26,30,32,42}$ Adjuvant therapy is limited for JOF. ${ }^{8}$ The use of adjuvant radiotherapy is controversial; while some case reports have described use of adjuvant proton radiotherapy for recurrent disease, ${ }^{28}$ others have argued that radiotherapy is ineffective and contraindicated due to concern for a potential increased risk of malignant transformation. ${ }^{33}$ Patients with JOF require long-term follow-up because of the high risk of recurrence. ${ }^{30,35}$

JTOFs can infiltrate surrounding bone and therefore excision of surrounding tissue (margins) is typically required for complete resection. ${ }^{13,42}$ Limited disease often does not require radical surgery, ${ }^{4,13,35}$ but larger, locally destructive, or recurrent lesions may necessitate en bloc resection or similarly aggressive surgical approaches. ${ }^{26,32}$ The challenges associated with resection of JOFs include hemorrhage secondary to neovascularization and injury to surrounding structure due to the adherent nature of the tumor. ${ }^{10} \mathrm{In}$ our case, surgical EBL was $6 \mathrm{~L}$, and the tumor was quite adherent to the dura of the sacral thecal sac, requiring me- ticulous dissection. It is known that sacral tumor resections can be associated with increased blood loss. The median EBL following en bloc resections of sacral tumors has been reported to be $3.9 \mathrm{~L},{ }^{16}$ and the lesions located cephalad to the S2-3 disc space were an independent risk factor for increased intraoperative EBL. ${ }^{41}$ Consideration of preoperative embolization may minimize blood loss. ${ }^{10}$

The surgical management of tumors involving the sacrum presents significant technical challenges, especially when managing aggressive, destructive, or malignant lesions..$^{20}$ In managing our patient, the first step in formulating a surgical plan was the decision as to pursue en bloc versus intralesional resection. En bloc resection via partial and total sacrectomy has been demonstrated to prolong progression-free survival and improve local control in both infiltrative benign and malignant tumors, ${ }^{31}$ but this approach is also associated with serious potential morbidity, including permanent lower-extremity sensory and motor deficits, sexual dysfunction, and bowel and bladder incontinence ${ }^{46}$ Complication rates in en bloc resection of spinal tumors are highest in caudal partial or total sacrectomies. ${ }^{48}$ While en bloc resection may be the preferred approach for recurrent and locally aggressive extraspinal JTOF, ${ }^{26,32}$ lesion proximity to vital structures, including the thecal sac, major vessels, and nerve roots, may preclude en bloc resection in a 29 -year-old patient. Furthermore, the improvement of local control conferred by en bloc resection has been shown to be attenuated in the context of recurrent sacral disease, although the literature still supports en bloc resection of recurrent lesions when feasible..$^{1,48}$ 
Per the Enneking staging system, benign tumors that are symptomatic and extend beyond the tissue compartment, such as in this case, are classified as aggressive (S3 ), and en bloc resection is recommended to reduce the potential for local recurrence. ${ }^{71}$ However, the risk of local recurrence must be weighed against the potential for serious iatrogenic neurological deficits. ${ }^{12}$ In some lesions, particularly locally aggressive benign lesions, Enneking Appropriate resection might entail unacceptable morbidity. ${ }^{3,22}$ After taking into consideration the patient's age, potential functionality, and tumor burden, it was decided that an en bloc resection via sacrectomy could result in unacceptable morbidity. Complete removal via intralesional resection with a nerve-sparing approach was thought to offer the patient the best overall outcome.

Following resection, the decision as to the plan for sacral reconstruction was not trivial. There is no consensus regarding the optimal method for lumbosacral reconstruction after resection of sacral tumors. ${ }^{9,34}$ Current recommendations encourage instrumentation in all but a very select number of patients to maintain spinopelvic stability, prevent sacral herniation, and limit the formation of an empty dead space at the site of resection. ${ }^{9,20,34} \mathrm{Sa}$ crectomy involving S-1 requires instrumentation to allow weight-bearing ability postoperatively. ${ }^{2}$ The options for lumbosacral reconstruction include spinopelvic fixation with pedicle and iliac screw-rod systems, posterior pelvic ring fixation (using transiliac bars, custom plates, structural allografts, prosthetic cages, or U-shaped rods), and anterior spinal column fixation (using iliolumbar screws, vertical rods, titanium mesh, expandable cages, or fibular grafts). ${ }^{5,9,45}$ Instrumentation may be further augmented via sacroplasty using MMA or fibular allografts. ${ }^{2,9}$ In our patient, posterior lumbopelvic fixation was achieved with double iliac screws bilaterally and a 4-rod technique. The sacrum was reconstructed directly onto the old corpectomy cage utilizing wires and MMA.

The delayed presentation of a left foot drop was quite unexpected on its own. Postoperative drains were left in place, and the patient was neurologically normal at discharge of index hospital admission. The organized hematoma found on reoperation was unusual, although resection of large sacral tumors have been shown to result in hematoma formation due to the resulting dead space..$^{24,27}$ Furthermore, isolated radicular symptoms secondary to compression by hematomas are uncommon, but rare cases have been reported in the literature. ${ }^{11,21,23}$ In each of these reported cases, elimination of the hematoma was associated with a substantial reduction in radicular symptoms. Given that the focal deficit (left foot drop) our patient experienced coincided with the location of the hematoma at the L-5 nerve root and that the patient experienced significant improvement in strength after hematoma evacuation, the hematoma was likely the precipitant of the new neurological findings.

The development of a delayed deep tissue infection has been documented in the past following sacral tumor resection, occurring in $8 \%-39 \%$ of published cases..$^{24,36}$ M. fortuitum is a rapidly growing nontuberculous $\mathrm{Myco-}$ bacterium that may cause wound, pulmonary, bone, joint, and surgical site infections. ${ }^{19,25}$ Surgical site infections due to M. fortuitum classically present, as in this case, with a long incubation period and delayed onset. ${ }^{19,25} \mathrm{M}$. fortuitum surgical site infections require surgical debridement and extended treatment with amikacin and clarithromycin or a fluoroquinolone..$^{25,43}$

Spinal JOFs are an exceptionally rare lesion, but they should be in the differential diagnosis of primary spinal tumors. After a histopathological diagnosis, the first line treatment of a JOF is resection. En bloc resection should be considered in patients with large, aggressive, or recurrent lesions. However, en bloc resection of locally aggressive but benign spinal or sacral lesions may confer unacceptable morbidity in a young patient. Intralesional resection allows for debulking of the tumor mass while sparing nerve roots. Potential morbidity associated with intralesional resection of large sacral lesions includes hematoma/ seroma formation, deep wound infection, and transient neurological deficits.

\section{References}

1. Ailon T, Torabi R, Fisher CG, Rhines LD, Clarke MJ, Bettegowda $\mathrm{C}$, et al: Management of locally recurrent chordoma of the mobile spine and sacrum: a systematic review. Spine (Phila Pa 1976) 41 (Suppl 20):S193-S198, 2016

2. Arıkan M, Togral G, Hasturk AE, Kekec F, Parpucu M, Gungor S: Management of sacral tumors requiring spino-pelvic reconstruction with different histopathologic diagnosis: evaluation with four cases. Asian Spine J 9:971-977, 2015

3. Bandiera S, Boriani S, Donthineni R, Amendola L, Cappuccio M, Gasbarrini A: Complications of en bloc resections in the spine. Orthop Clin North Am 40:125-131, vii, 2009

4. Banu K, Palikat SM: Juvenile trabecular ossifying fibroma of the mandible. J Maxillofac Oral Surg 9:87-90, 2010

5. Bederman SS, Shah KN, Hassan JM, Hoang BH, Kiester PD, Bhatia NN: Surgical techniques for spinopelvic reconstruction following total sacrectomy: a systematic review. Eur Spine J 23:305-319, 2014

6. Bohn OL, Kalmar JR, Allen CM, Kirsch C, Williams D, Leon ME: Trabecular and psammomatoid juvenile ossifying fibroma of the skull base mimicking psammomatoid meningioma. Head Neck Pathol 5:71-75, 2011

7. Boriani S, Weinstein JN, Biagini R: Primary bone tumors of the spine. Terminology and surgical staging. Spine (Phila Pa 1976) 22:1036-1044, 1997

8. Breheret R, Jeufroy C, Cassagnau E, Malard O: Juvenile ossifying fibroma of the maxilla. Eur Ann Otorhinolaryngol Head Neck Dis 128:317-320, 2011

9. Choi MK, Jo DJ, Kim SB: Pelvic reconstruction surgery using a dual-rod technique with diverse U-shaped rods after posterior en bloc partial sacrectomy for a sacral tumor: 2 case reports and a literature review. World Neurosurg 95:619.e11-619.e18, 2016

10. Ciniglio Appiani M, Verillaud B, Bresson D, Sauvaget E, Blancal JP, Guichard JP, et al: Ossifying fibromas of the paranasal sinuses: diagnosis and management. Acta Otorhinolaryngol Ital 35:355-361, 2015

11. Daureeawoo R, Baliga S, Mohahmed W, Ahmed EB: A case report of an unusual cause of postoperative leg pain after posterior lumbar fusion. Ann Med Surg (Lond) 19:29-32, 2017

12. Dea N, Gokaslan Z, Choi D, Fisher C: Spine oncology - primary spine tumors. Neurosurgery 80 (3S):S124-S130, 2017

13. El-Mofty S: Psammomatoid and trabecular juvenile ossifying fibroma of the craniofacial skeleton: two distinct clinicopathologic entities. Oral Surg Oral Med Oral Pathol Oral Radiol Endod 93:296-304, 2002

14. El-Mofty SK, Nelson BL, Toyosawa S: Ossifying fibroma, in El-Naggar AK, Chan JKC, Grandis JR, et al (eds): WHO 
Classification of Head and Neck Tumours, ed 4. Lyon: IARC Press, 2017, pp 251-252

15. Enneking WF, Spanier SS, Goodman MA: A system for the surgical staging of musculoskeletal sarcoma. Clin Orthop Relat Res (153):106-120, 1980

16. Fourney DR, Rhines LD, Hentschel SJ, Skibber JM, Wolinsky JP, Weber KL, et al: En bloc resection of primary sacral tumors: classification of surgical approaches and outcome. J Neurosurg Spine 3:111-122, 2005

17. Jaafari-Ashkavandi Z, Shirazi MY, Assar S: Desmoplastic fibroblastoma in maxillary alveolar bone mimicking an odontogenic lesion: a novel case report with review of literature. Turk Patoloji Derg [epub ahead of print], 2017

18. Johnson LC, Yousefi M, Vinh TN, Heffner DK, Hyams VJ, Hartman KS: Juvenile active ossifying fibroma. Its nature, dynamics and origin. Acta Otolaryngol Suppl 488:1-40, 1991

19. Kalita JB, Rahman H, Baruah KC: Delayed post-operative wound infections due to non-tuberculous Mycobacterium. Indian J Med Res 122:535-539, 2005

20. Kiatisevi P, Piyaskulkaew C, Kunakornsawat S, Sukunthanak B: What are the functional outcomes after total sacrectomy without spinopelvic reconstruction? Clin Orthop Relat Res 475:643-655, 2017

21. Kleiner JB, Donaldson WF III, Curd JG, Thorne RP: Extraspinal causes of lumbosacral radiculopathy. J Bone Joint Surg Am 73:817-821, 1991

22. Lador R, Bandiera S, Gasbarrini A, Ghermandi R, Boriani S: Treatment of spinal tumors in a high volume center has direct impact on local recurrence, morbidity, and mortality. Clin Spine Surg 30:E1074-E1081, 2017

23. Lakkol S, Sarda P, Karpe P, Krishna M: Conservative management of psoas haematoma following complex lumbar surgery. Indian J Orthop 48:107-110, 2014

24. Li D, Guo W, Qu H, Yang R, Tang X, Yan T, et al: Experience with wound complications after surgery for sacral tumors. Eur Spine J 22:2069-2076, 2013

25. Madhusudhan NS, Malini A, Sangma MM: A case of surgical site infection caused by mycobacterium fortuitum, following herniorrhaphy. J Clin Diagn Res 10:DD01-DD02, 2016

26. Manjunatha BS, Das N, Naik S, Gowramma R: Trabecular variant of juvenile aggressive ossifying fibroma of anterior mandible. Pediatr Rep 4:e24, 2012

27. Maricevich M, Maricevich R, Chim H, Moran SL, Rose PS, Mardini S: Reconstruction following partial and total sacrectomy defects: an analysis of outcomes and complications. J Plast Reconstr Aesthet Surg 67:1257-1266, 2014

28. Neidert MC, Woernle CM, Burkhardt JK, Reimann R, Hug E, Bernays RL: Case report: trabecular juvenile ossifying fibroma presenting as a sellar mass. J Neurol Surg A Cent Eur Neurosurg 74:405-409, 2013

29. Ohyama T, Ohara S, Momma F, Moto A, Nakata Y: Ossifying fibroma of the thoracolumbar spine: a case report and review of the literature. Surg Neurol 37:231-235, 1992

30. Osunde O, Iyogun C, Adebola R: Juvenile aggressive ossifying fibroma of the maxilla: a case report and review of the literature. Ann Med Health Sci Res 3:288-290, 2013

31. Palejwala AH, Fridley JS, Garcia K, Vasudevan SA, Khechoyan D, Rednam S, et al: Hemisacrectomy with preservation of the contralateral sacral nerve roots and sacroiliac joint for pelvic neurofibrosarcoma in a 7-year-old child: case report with 2-year follow-up. J Neurosurg Pediatr 19:102107,2017

32. Rahman T, Hashmi GS, Ansari H: Trabecular variant of juvenile aggressive ossifying fibroma. Rare Tumors 7:5890, 2015

33. Rai S, Kaur M, Goel S, Prabhat M: Trabeculae type of juvenile aggressive ossifying fibroma of the maxilla: Report of two cases. Contemp Clin Dent 3 (Suppl 1):S45-S50, 2012

34. Reynolds JJ, Khundkar R, Boriani S, Williams R, Rhines LD,
Kawahara N, et al: Soft tissue and bone defect management in total sacrectomy for primary sacral tumors: a systematic review with expert recommendations. Spine (Phila Pa 1976) 41 (Suppl 20):S199-S204, 2016

35. Rinaggio J, Land M, Cleveland DB: Juvenile ossifying fibroma of the mandible. J Pediatr Surg 38:648-650, 2003

36. Ruggieri P, Angelini A, Pala E, Mercuri M: Infections in surgery of primary tumors of the sacrum. Spine (Phila Pa 1976) $37: 420-428,2012$

37. Shaik M, Kishore Babu T, PrabhuSekhar G, Venkata Ramana Murthy V: Juvenile ossifying fibroma of the maxilla: a case report. J Maxillofac Oral Surg 14 (Suppl 1):69-72, 2015

38. Skinner HR, Vargas A, Solar A, Foncea C, Astorga P: Desmoplastic fibroma of the mandible in a pediatric patient: a case report of resection and reconstruction with a six-year followup. J Oral Maxillofac Surg 75:1568.e1-1568.e10, 2017

39. Slootweg PJ: Juvenile trabecular ossifying fibroma: an update. Virchows Arch 461:699-703, 2012

40. Slootweg PJ, Müller H: Juvenile ossifying fibroma. Report of four cases. J Craniomaxillofac Surg 18:125-129, 1990

41. Tang X, Guo W, Yang R, Tang S, Ji T: Risk factors for blood loss during sacral tumor resection. Clin Orthop Relat Res 467:1599-1604, 2009

42. Thankappan S, Nair S, Thomas V, Sharafudeen KP: Psammomatoid and trabecular variants of juvenile ossifying fibroma-two case reports. Indian J Radiol Imaging 19:116-119, 2009

43. Tsai SC, Chen LH, Liao HH, Chiang CY, Lin WL, Chen SC, et al: Complicated skin and soft tissue infection with Mycobacterium fortuitum following excision of a sebaceous cyst in Taiwan. J Infect Dev Ctries 10:1357-1361, 2016

44. Uslu H, Cakir E, Arslan E, Ersoz S, Baykal S: Ossifying fibroma of the lumbar spine: case report. Turk Neurosurg 21:104-106, 2011

45. Varga PP, Szoverfi Z, Lazary A: Surgical resection and reconstruction after resection of tumors involving the sacropelvic region. Neurol Res 36:588-596, 2014

46. Verlaan JJ, Kuperus JS, Slooff WB, Hennipman A, Oner FC: Complications, secondary interventions and long term morbidity after en bloc sacrectomy. Eur Spine J 24:2209-2219, 2015

47. Waldron CA: Fibro-osseous lesions of the jaws. J Oral Maxillofac Surg 51:828-835, 1993

48. Yamazaki T, McLoughlin GS, Patel S, Rhines LD, Fourney DR: Feasibility and safety of en bloc resection for primary spine tumors: a systematic review by the Spine Oncology Study Group. Spine (Phila Pa 1976) 34 (22 Suppl):S31-S38, 2009

\section{Disclosures}

Dr. Ames: employee of UCSF; consultant for DePuy Synthes, Medtronic, Stryker, K2M, and Medicrea; and royalties from Stryker, Biomet Spine, DePuy Synthes, and NuVasive.

\section{Author Contributions}

Conception and design: Ames, Lau. Acquisition of data: Dalle Ore, Lau, Davis. Analysis and interpretation of data: Dalle Ore, Lau, Davis. Drafting the article: Dalle Ore, Lau, Davis. Critically revising the article: Ames, Dalle Ore, Lau, Safaee. Reviewed submitted version of manuscript: Ames, Dalle Ore, Lau, Safaee. Study supervision: Ames.

\section{Correspondence}

Christopher P. Ames: University of California, San Francisco, CA. amesc@neurosurg.ucsf.edu. 